\title{
Benefits and Barriers to Using Epidemiology Data in Environmental Risk Assessment
}

\author{
Kathleen C. Raffaele*, Suryanarayana V. Vulimiri and Thomas F. Bateson
}

\author{
National Center for Environmental Assessment, Office of Research and Development, U.S. Environmental Protection \\ Agency, Washington, DC 20460, USA
}

\begin{abstract}
Over the past three decades, a formal risk assessment process has been developed to provide consistent and transparent methods for the assessment of potential human health risks from exposure to environmental chemicals. Given a focus on risk to human health, epidemiological studies that identify associations between exposure to environmental chemicals and adverse health effects in humans have the potential to provide critically important information to this process. For many chemicals, however, available epidemiology studies have been found to have limited utility in informing human health risk assessments. In order to investigate this paradox, we have used several case examples to explore the utility of various types of epidemiological data in informing key elements of the risk assessment process (hazard identification, exposure-response assessment, and exposure assessment). Examples from the epidemiologic literature on environmental chemicals are used to illustrate the issues that arise in using available studies for various types of chemical risk assessments. The case examples illustrate several advantages in using epidemiology data, but also identify a number of barriers to its use, frequently related to limitations in exposure assessment. The examples also highlight ways in which the utility of both toxicology and epidemiology data can be enhanced by considering the data in combination, and integrating the results across study categories. Recent scientific developments offer hope for improving the utility of both types of data, and thus enhancing the reliability of future risk assessment efforts.
\end{abstract}

Keywords: Epidemiology, risk assessment, pesticides, dose-response assessment.

\section{INTRODUCTION}

In 1983, the National Research Council (NRC) developed a formal conceptual framework for human health risk assessment in its report "Risk Assessment in the Federal Government: Managing the Process" (otherwise known as the 'Red Book' [1]). In that framework, NRC defined risk assessment as "the qualitative or quantitative characterization of the potential health effects induced by particular substances in individuals or populations." This framework was widely accepted and endorsed by the U.S. Environmental Protection Agency (USEPA) soon after its release (as discussed in [2]) and over the years EPA and other organizations have developed risk assessment guidelines to ensure that risk assessments are performed in a consistent and scientifically sound manner [2-11]. Within EPA the risk assessment process has evolved over the past several decades from when the EPA conducted its first formal risk assessment in 1975, as described in "Risk Assessment Principles and Practice" [2]. In recent years, NRC has published additional reports related to risk assessment issues; these reports have expanded on the issues discussed in the original framework (for example, doseresponse evaluation and use of uncertainty factors), and addressed ways to incorporate new issues and scientific advances into the risk assessment process $[12,13]$.

*Address correspondence to this author at the National Center for Environmental Assessment, Office of Research and Development, U. S. Environmental Protection Agency, Mail Code 8623P, 1200 Pennsylvania Ave. NW, Washington, DC 20460, USA; Tel: 703-308-7255; Fax: 703-3478692; E-mail: raffaele.kathleen@epa.gov
According to the 'Red Book' framework, risk assessment is divided into four steps: i) hazard identification, ii) doseresponse assessment; iii) exposure assessment, and iv) risk characterization. In each of the first three steps, the risk assessment assembles the available data, and identifies its strengths and limitations; in the fourth step, the information from the first three steps is synthesized.

The information available for performing human health risk assessment varies widely among chemicals, and may include studies performed using in vitro systems (including prokaryotes and lower eukaryotes), in vivo systems such as animal models, or various types of human data, including epidemiology studies of exposed populations. Data generated through epidemiology studies may potentially be used to inform any or all of the first three components of the risk assessment process (hazard identification, dose-response assessment, and exposure assessment) identified by the NRC [14-17]. In the development of human health risk assessments, data evaluating health effects of the chemical of concern in humans are generally preferred over information obtained using in vivo animal studies or in vitro studies, since human epidemiology studies directly assess health effects on the population of concern at exposure levels that are directly relevant to people. Use of human data eliminates the need to address several types of uncertainties inherent in the use of animal data, including uncertainties regarding cross-species extrapolation and differences in exposure paradigms (potentially including the route, duration, and level of exposures) between typical animal studies and expected human exposures [14]. 
Swaen [18] and others [16] have written on the use of epidemiologic data in risk assessment and highlighted some of the advantages and limitations. It is well recognized that the greatest limitation in epidemiological data is accurate exposure assessment, an issue that is often exacerbated by the retrospective ascertainment of data after an outcome of interest has occurred. Exposure assessment is most challenging in case-control studies and retrospective cohort studies, but influences all observational studies. It is also well understood that when any exposure measurement error is random or non-differential, any resulting bias (due to that error) in associated effect estimates is typically towards the null, yielding weaker effects than might otherwise have been observed in the absence of error. In addition to measurement error, another exposure-related limitation of some occupational epidemiology studies is the limited size of the population that may have been exposed to a particular chemical, which can diminish statistical power so much as to preclude the statistical detection of any potential adverse effect [18].

Other limitations of epidemiologic data from a risk assessment perspective include the restriction to health effects that have already occurred and the corollary issue of long latencies of many cancers that may hamper the timeliness of epidemiology evidence collection in support of risk assessment [19]. It is also true that, in addition to exposure measurement error (information bias), other potential biases such as selection bias and confounding may be present and may influence epidemiologic findings.

While these issues may appear daunting for the purposes of the application of epidemiologic results to the risk assessment process, observations based on epidemiology studies have several potent strengths that warrant their careful consideration. First among them is that these data directly describe the human experience of exposure, often taking into consideration typically relevant co-exposures, such as other occupational and environmental exposures that may cause morbidity or mortality, behavioral characteristics such as diet, smoking habits, medication and alcohol consumption [19], and personal characteristics such as age, gender, socio-economic status, body mass index, etc. Swaen [18] highlights a useful example showing that observational studies of people in the natural environment can reveal very important risks that would have been missed in animal studies. Swaen [18] cites the work of Steenland and Thun [20] who demonstrated the multiplicative effect of smoking and asbestos on the relative risk of lung cancer and makes the statement that "because of this interaction animal studies investigating the risks of asbestos exposure in absence of exposure to cigarette smoke would lead to an underestimation if extrapolated to smoking humans" [18].

An additional strength of epidemiology studies is the inclusion in study populations of sensitive individuals or sensitive population subgroups that may reveal particular susceptibility to an exposure of concern. Also, the exposure levels of the study populations are most likely to be within the relevant ranges of concern to risk assessors evaluating human environmental exposures [19], eliminating the need for high to low dose extrapolation which is frequently required when using animal toxicology data in human health risk assessments for environmental chemicals.
While these strengths and limitations are self evident, our work of applying toxicological and epidemiological data in quantitative risk assessment has led to the elucidation of several additional decision points that have not been well described. In the face of the differences in the type of information available from animal toxicity, in vitro toxicity, and epidemiology studies, it is useful to consider the strengths and limitations of these types of information in conducting quantitative risk assessments. These issues go beyond questions of whether epidemiologic data can be used for risk assessment to focus on whether, and under what circumstances, epidemiological data should be used when competing toxicological data may also be available.

In developing a risk assessment for environmental chemicals, the weight of the available epidemiologic evidence is carefully considered in characterizing the hazard, and the potential for the observed results to be the result of bias or chance must be ruled out prior to the determination of a causal relationship between exposure and outcome. In each risk assessment that considers the use of epidemiologic data for quantitative risk assessment, the accuracy and reliability of the reported findings in human epidemiologic studies must be weighed against that which can be garnered from the use of animal studies with the application of traditional uncertainty factors.

In order to elucidate the ways in which different types of data can best contribute to human health risk assessment for environmental chemicals, it is useful to discuss the strengths and limitations of epidemiology data in supporting specific components of human health risk assessments. Advantages of using available epidemiology data, as well as some of the barriers to its use, are illustrated and discussed in the context of case examples in which: 1) available epidemiological data was of very limited utility for hazard identification or for dose-response assessment; 2) available epidemiological data were useful for hazard identification, but interpretation in the context of available biological information challenged the extent of that utility for risk quantification; 3) available epidemiological data were sufficient to inform the risk assessment, including both hazard identification and risk quantification.

\section{CASE EXAMPLES}

\section{Example 1. Contribution of Pesticide Exposure to Parkinson's Disease Risk}

Parkinson's disease is a chronic human neurodegenerative disease, characterized (at least initially) by motor system dysfunction resulting in bradykinesia, tremors, and other neurological symptoms [21]. Although some hereditary cases have been identified, most cases of Parkinson's disease are idiopathic, i.e., the proximate cause for the individual case is unknown. Pathologically, Parkinson's disease is characterized by degeneration in specific brain regions (e.g., the substantia nigra). Degeneration of the same brain regions was also identified following exposure to 1-methyl-4-phenyl-1,2,3,6tetrahydropyridine (MPTP), which was a contaminant of 1methyl-4-phenyl-4-propiopiperidine (MPPP), a street analog of the narcotic meperidine [21,22]. A series of animal studies identified a mechanism of action for the neurodegenerative effects of MPTP; MPPP is metabolized to form MPP+, 
which is specifically taken up by dopaminergic neurons in the substantia nigra, where it then acts as a mitochondrial poison (inhibiting mitochondrial electronic transport chain complex I). Several pesticides (including rotenone, pyridaben, and paraquat) are known to interfere with the function of mitochondrial complex I [23], and others (for example, manganese-containing pesticides such as maneb) also have toxic effects on nervous system function [24]. Thus, it has been postulated that environmental exposure to pesticides may be associated with an increased risk for Parkinson's disease. A variety of animal models have been developed to investigate this hypothesis, some of which appear to substantiate an association between (e.g.) rotenone exposure [25] or a combination of paraquat and maneb [24] and brain or behavioral changes similar to those seen in humans with Parkinson's disease.

Multiple epidemiology studies have also evaluated possible associations between pesticide exposure and an increased risk of Parkinson's disease. However, the specific findings vary considerably across studies, with inconsistent findings for individual pesticide chemicals or chemical groupings (for example, increased risk, decreased risk, or no difference in risk for Parkinson's disease have all been found associated with herbicide exposure; as reviewed in [26]).

This large body of epidemiological literature, with inconsistent findings regarding associations between pesticide exposure and Parkinson's disease in exposed populations, illustrates some of the difficulties in utilizing epidemiological data for hazard identification. Differences in findings among studies may be related to the use of broad exposure categories (pesticides and their formulations vary considerably in their chemical class, use, and likely health effects). It is also likely that there may be limitations in the methods used to identify and describe exposed populations. As one example, pesticide-exposed subjects may be defined as individuals living on farms, or those who were employed in a specific job category [26,27]. Exposures to individuals who would be included as subjects under these two classifications are likely quite different. In addition, the use of broad categories of exposure may result in misclassification of subjects, obscuring any pesticide-disease association. Several recent studies using records of pesticide applications proximate to subjects' residences illustrate an effort to develop more precise exposure information [28-30]; this type of information has allowed for greatly improved evaluation regarding the likelihood of exposure to particular pesticide chemicals (i.e., greater certainty that exposure did or did not occur), but quantitative exposure (i.e., 'dose') estimation was not possible, and exposure routes likely varied, based (for example) on whether or not drinking water was primarily obtained from local wells [28].

Another potential contributor to the disparate findings among studies is the generic categorization of pesticide exposure (i.e., classifying subjects as pesticide-exposed, without regard to the specific pesticide chemical). Pesticides vary considerably in their structure and mode of action, and farmers and farm workers are rarely exposed to a single pesticide. Since it is likely that increased risk for Parkinson's disease would be associated only with individual chemicals or specific combinations of chemicals, use of broad exposure categories may obscure relationships with individual chemicals, again decreasing the likelihood of finding an association. Evaluation of association between Parkinson's disease and particular chemicals or combinations of chemicals has resulted in positive associations in some studies but not in others; in addition, associations identified in some studies are not always seen in other studies evaluating the same chemicals [28-34].

Use of dichotomous exposure categories (i.e., 'ever' versus 'never' exposed) may also obscure chemical-disease relationships, as it is possible that adverse effects might be seen only with exposure above some 'threshold' level or for some 'threshold' duration; detailed exposure evaluations that provide quantitative levels of exposure or durations of exposure (which may be difficult to obtain) may be necessary for the causal association to be manifested, but are very difficult to obtain in most epidemiologic studies. In addition, available evidence indicates that Parkinson's disease is likely caused by multiple factors, including combinations of exposure and genetic susceptibility $[21,30,35]$. Although these types of factors are often difficult to evaluate in epidemiological studies, a recent study has found positive associations between increased risk for Parkinson's disease and combinations of genetic susceptibility, age at exposure, and exposure to specific pesticides [30].

This example illustrates several types of barriers encountered in developing epidemiological data to evaluate associations between diseases of complex etiology and exposures which are also complex (and difficult to measure). Evaluation of Parkinson's disease risk based on animal exposure models is not straightforward, given that behavior or pathology that might be indicative of Parkinson's disease is rarely seen and may not be adequately assessed in the animal models commonly used to assess toxicity of environmental chemicals (e.g., studies performed according to testing guidelines used by regulatory agencies [36]). Although strong epidemiological findings would thus contribute critical information to risk assessments for these chemicals, the barriers described above (and in particular the problems with accurately assessing exposure, including both the identification of the pesticide involved and the level and pattern of exposure) have greatly limited the utility of currently available epidemiological data in assessing the contribution of pesticide exposure to Parkinson's disease risk.

\section{Example 2. Developmental Neurotoxicity of Chlorpyrifos}

Over the past several years animal studies have generated data supporting the occurrence of developmental neurotoxicity following perinatal exposure to an organophosphate pesticide, chlorpyrifos [37]. Although the primary pesticidal mode of action for chlorpyrifos is the inhibition of an enzyme, acetylcholine esterase (resulting in a buildup of the neurotransmitter, acetylcholine, in the synaptic cleft), the mechanism for its neurodevelopmental effects has not been definitively identified. Effects have, however, been observed in multiple different animal models of brain development, both in vivo and in vitro [37,38]. Recently, several epidemiological studies have investigated whether developmental neurotoxicity could be detected in human infants exposed to chlorpyrifos during gestation [39- 
43]. These data have recently been reviewed, both in the literature [37] and by an EPA Scientific Advisory Panel (SAP) [44]. Both reviews concluded that, taken together, the human data raised serious concerns regarding developmental neurotoxicity of chlorpyrifos in humans. However, the SAP concluded that the epidemiologic data were not sufficiently robust to determine the exposure-response relationship for the human neurodevelopmental effects, even though the studies included measurement of chlorpyrifos and/or its metabolites in maternal tissue (blood, cord blood, or urine) and/or measurement of chlorpyrifos in the home during the gestation period.

Both of the above reviews noted specific concerns that limited the use of results of the epidemiology studies for quantitative risk assessment, for example: (1) limited exposure assessments (at one or a few time points) were not sufficient to provide a good understanding of exposure over the entire period of concern, and did not allow assessment of possible peak exposure levels; this was of particular concern given the relatively short half-life of chlorpyrifos and its metabolites, and the developmental nature of the effects of concern; (2) although effects of concern were seen in several studies, the specific findings varied across studies, making it difficult to compare results; (3) concomitant exposure to other substances, including other pesticides, made it difficult to attribute the effects specifically to chlorpyrifos exposure. Indeed, although the measured exposure levels raised the possibility that developmental neurotoxicity was occurring at doses below those causing acetylcholine esterase inhibition (the pesticidal mode of action for chlorpyrifos), the review panel viewed the limitations of the exposure information to be sufficiently large that they were unwilling to conclude that regulatory levels based on preventing cholinesterase inhibition would not be protective of developmental neurotoxicity, instead proposing that the studies be used qualitatively as a source of uncertainty in the risk assessment for these effects $[37,44]$.

The foregoing example highlights several barriers to the use of epidemiology data in quantitative risk assessment (dose-response assessment):

(a) Limitations on exposure information, including single or few assessments of specific exposure levels during a long term exposure to shifting levels of the substance of concern. In the absence of complete exposure information, it is often not possible to determine whether the effects of concern are related to peak levels of exposure, duration of exposure, or some combination thereof.

(b) Difficulty in isolating dose-response relationships to one chemical in situations where multiple exposures are occurring simultaneously. Unless detailed exposure information is available that allows separate determination of exposure for the various compounds, it may be difficult to control for potentially confounding co-exposures.

(c) Problems in understanding the relationship between the biomonitoring data (often a metabolite or biomarker such as enzyme inhibition or DNA adducts) and the exposure levels to the substance of concern. This is particularly problematic in cases where there may also be direct exposure to the metabolite, as is the case for chlorpyrifos [37], and where the biological half-life of the compound is short (limiting the period during which relevant exposures can be assessed). Unless the relationship between the biomonitoring data and the exposure can be determined, it is difficult or impossible to understand the dose-response relationship between the adverse health effect and specific exposure levels.

Although these limitations in the available epidemiology data for chlorpyrifos were seen as sufficient to preclude the use of quantitative exposure information to derive a point-ofdeparture for use in risk assessment, the chlorpyrifos epidemiology studies were considered very useful in supporting the human relevance of findings in multiple animal studies, and in that role were useful in informing the human health risk assessment. Indeed, the SAP concluded that consideration of the human and animal data together led to the conclusion that "maternal chlorpyrifos exposure would likely be associated with adverse neurodevelopmental outcomes in humans" [44]. In the absence of the human epidemiological studies, it is unlikely that such a strong conclusion would have been reached.

\section{Example 3. Skin Lesions and Skin Cancer from Inorganic Arsenic}

The entire database of EPA's Integrated Risk Information System (IRIS) health assessments was reviewed through June 2007 by Persad and Cooper [14]. They catalogued 44 assessments, out of a total of 545 assessments in the database, which had used human data to derive non-cancer or cancer risk estimates. Predominantly, the epidemiologic data were used to support non-cancer reference concentrations or reference doses (RfD). In only 12 assessments did they find that human data (from clinical studies, environmental and occupational epidemiology studies) were used to derive inhalation unit risks for cancer and just three chemical assessments calculated cancer slope factors based on epidemiologic data [14]. Their analysis [14] provides an excellent historical example of the benefits and barriers to using epidemiologic data from the 1988 IRIS assessment of inorganic arsenic. The example of inorganic arsenic is provided only to illustrate the past use of epidemiological data for deriving risk values and is not intended as a commentary on, or judgment of, past work, nor is it an attempt to reexamine those historical decisions in a modern context.

Inorganic arsenic is one of only three chemicals in the IRIS database that derived an inhalation unit risk and a cancer slope factor from human epidemiologic data. In the 1988 assessment, an RfD was also derived based on the studies by Tseng and coworkers $[45,46]$ which reported on the incidence and prevalence of blackfoot disease among more than 40,000 residents in an area of southwestern Taiwan [47]. Blackfoot disease is a peripheral vascular disorder resulting in gangrene of the extremities. A lowestobserved-adverse-effects level (LOAEL) was defined among the group of people who were highly exposed $(170 \mu \mathrm{g} / \mathrm{L})$ and the control group was defined as a no-observed-adverseeffects level or NOAEL $(9 \mu \mathrm{g} / \mathrm{L})$. 
In 1988, confidence in this NOAEL was considered medium as the control group showed no evidence of skin lesions and, EPA assumed at the time, no evidence of blackfoot disease [47], although the latter point was not explicitly stated by Tseng and coworkers $(1968,1977)$. The incidence of skin lesions increases sharply in individuals above 20; the incidence of blackfoot disease increases sharply in individuals above 40 (See figures 5, 6 and 7of ref. [46]:). Although the control group contained 2552 individuals, only 957 (approximately 38\%) were older than 20, and only 431 (approximately 17\%) were older than 40. Since the control individuals were so young, they were likely to have been at low background risk of adverse effects, so it could not be known in 1988 whether $9 \mu \mathrm{g} / \mathrm{L}$ was truly a NOAEL or whether those individuals might have developed lesions and blackfoot disease as they aged into the higher risk age group. This uncertainty reduced confidence in the RfD [47]. Although there are some limitations associated with this study, it was considered to be the most powerful study available on arsenic exposure to people at the time of the 1988 assessment and the IRIS assessment relied upon the human epidemiologic data for the RfD [47].

Likewise for the cancer assessment, the IRIS assessment relied on the human epidemiologic data from the studies of Tseng and coworkers [45,46] to derive the inhalation unit risk and cancer slope factor (measures of cancer potency). The studies of over 40,000 Taiwanese exposed to arsenic in drinking water found significant excess skin cancer prevalence in comparison to 7500 residents of Taiwan and Matsu who consumed relatively arsenic-free water (Tseng et al., 1968; Tseng, 1977) [47]. The 1988 IRIS assessment reported that there was sufficient evidence from epidemiologic data to classify inorganic arsenic as a human carcinogen, while noting the dietary differences between Taiwanese and U.S. populations, the potential differences in the underlying background rates of cancer, the possibility for the population's additional exposure to inorganic arsenic from sources other that in drinking water, and the potential for confounding by other contaminants in drinking water [47].

EPA did evaluate the available data at that time regarding carcinogenicity of inorganic arsenic in animals and found it to be inadequate, as carcinogenicity had not been consistently demonstrated in several species of test animals administered arsenic by different routes [47]. Specifically, the IRIS assessment cited a paper by Furst [48], who reviewed carcinogenicity studies of nine inorganic arsenic compounds in multiple strains of mice and rats, as well as in dogs, rabbits, swine and chickens. The studies reviewed by Furst [48] included exposure by the oral, dermal, inhalation and parenteral routes and all oxidative states of arsenic were tested. The EPA cited [47] the conclusion of Furst [48] that none of the animal studies demonstrated the carcinogenicity of arsenic and noted that animals may not be an appropriate model for the carcinogenicity of arsenic in humans.

The EPA based qualitative conclusions and quantitative risk values on the human epidemiologic data [47] which directly described the exposure experience of more than 40,000 people in their own environment.

\section{DISCUSSION}

The above case examples serve to identify a number of important benefits to be gained by using epidemiology data in risk assessment, e.g.: 1) Identification of specific human health effects that were not identified in animal models (e.g., inorganic arsenic); 2) Affirmation of the human relevance of effects identified in animal models (e.g., neurodevelopmental effects seen with chlorpyrifos); 3) Ability to evaluate health effects for which animal models are unavailable or limited (e.g., inorganic arsenic and cancer, pesticides and Parkinson's disease); 4) Evaluation of health effects in the range of expected human exposures (e.g., inorganic arsenic); 5) Utility of biomonitoring information (chlorpyrifos).

In addition to the identified benefits, a number of important barriers were also identified, e.g.: 1) Difficulty in converting human biomonitoring information to exposure levels that can be confidently associated with a specific hazard (difficulties related both to back-calculating from measured tissue levels to exposure levels as well as to understanding the source of measured metabolites [i.e. direct exposure to metabolite versus products of internal metabolism], chlorpyrifos); 2) Difficulty in identifying exposed populations (both with respect to identifying the specific compounds to which exposure occurred and which individuals were exposed; Parkinson's disease and pesticides); 3) Difficulty in isolating specific exposures (both routes of exposure and quantification of exposure; all three examples); 4) Concerns regarding appropriateness of evaluated population (in the key arsenic studies, including diet, genetics, alternative exposure sources and the issue of the younger age of the control group).

In addition to these specific findings, the case studies also provide useful illustrations of the ways in which toxicology data and epidemiology data can be used in combination, both to identify and understand health effects of concern, as well as to guide future research. For both the 'Parkinson's disease and pesticides' and the chlorpyrifos case examples, toxicology studies (utilizing data from both in vivo and in vitro evaluations) have provided biological information (mechanistic hypotheses, kinetic models, metabolic information, etc.) that has enabled epidemiologists to focus their studies on (e.g.) particular endpoints (chlorpyrifos and neurodevelopmental effects) or particular types of exposure (Parkinson's disease and specific pesticides or combinations of pesticides). Conversely, the epidemiology findings regarding neurodevelopmental effects of chlorpyrifos exposure provided strong support for earlier findings in animal models that had, in the absence of supporting human data, been considered much less compelling. As illustrated by these case examples, the combination and integration of information from both toxicology and epidemiology studies can frequently lead to a much stronger, more scientifically sound risk assessment.

Utility of information in support of quantitative risk assessment is often viewed through the lens of an individual reviewer's scientific training. From the viewpoint of the toxicologist, data is usually evaluated from the perspective 
of its ability to inform the toxicologist's 'Prime Directive': Paracelsus' assertion that 'the dose makes the poison'. Relevant corollaries of this founding doctrine are: 1) both the type and magnitude of effect following exposure to a given substance are entirely dependent upon the exposure level (dose); and 2) any substance may cause toxicity if the exposure level is high enough. Adherence to these 'basic principles' frequently makes it difficult for toxicologists to perceive the utility of epidemiologic data, where the available exposure information is usually much more limited than what they see in a typical toxicology study using controlled exposure to experimental animals.

Epidemiologists also view data through their own perspective, where the focus is frequently on whether or not the available data are sufficiently strong to support a causal association between exposure to a specific chemical and a specific population-based health outcome. With respect to exposure assessment, multiple potential confounding exposures must often be evaluated, in addition to the chemical of concern, in order to document the specificity of the association. In many situations, precise exposure estimates are difficult to obtain, given the large variation in exposure both among individuals in the population and for a given individual across time. Accepting the greater probability that exposure misclassification will lead to null findings than false positive findings, relatively broad exposure ranges are often combined into single groupings, in order to increase the power of the study. With respect to the types of health effects evaluated, epidemiologists must often focus on a relatively narrow spectrum of potential effects, either in order to avoid false positive findings due to inclusion of large numbers of potential associations, or because of study design issues (e.g., case-control studies usually focus on single health effect categories). This type of design may provide greater precision in determining whether or not there is a relationship between an exposure and a specific type of health effect, but may not be able to evaluate the broader spectrum of effects that any given chemical may cause (and that are at least nominally evaluated in typical 'general' toxicity studies).

As illustrated in the case examples above, the utility of epidemiology data will vary considerably, dependant both on specific characteristics of the available studies and on other information that may be available about the chemical being evaluated. For some chemicals, limitations on exposure information may support only a qualitative use; while for others, differences in animal/human responses may require the use of human information for specific endpoints. In between those extremes lie many intermediate scenarios, where epidemiological data may support concern for certain types of effects, provide information regarding human relevance, or aid in the evaluation of the relative sensitivity of various endpoints in animals and humans. Increased efforts on the part of both toxicologists and epidemiologists will improve the integration of these various data sources, ultimately leading to improvements in the quality of human health risk assessments.

Recent scientific advances have also led to improved methodology that will help bridge the gap between the utility of information from animal and human studies. Improved analytical methods have allowed more accurate quantification of exposure, at lower exposure levels. Identification of biomarkers of exposure (e.g., metabolites, protein adducts, etc.) as well as of effect (e.g., enzyme inhibition, transcriptomics, etc.) have enhanced both the ability to quantify exposure (in humans and in animals) and to understand the relationship between responses of animals and humans to a given chemical exposure. Improved methods for banking tissues (e.g., from prospective epidemiological studies) have provided opportunities for retrospectively evaluating exposures or analyzing biomarkers in well-studied population cohorts, as well as in monitoring the exposure over extended time periods. Recent large cohort studies have focused on improved exposure assessment with the inclusion of a broader spectrum of endpoints. More widespread availability of these types of information will provide common ground for epidemiologists and toxicologists to improve quantitative risk assessment.

In addition to the wider availability of more quantitative data, inclusion of more detailed exposure or biomarker information in published studies could increase the utility of those studies for quantitative risk assessment. Publication of individual data or more detailed exposure/response information (including as supplemental files) will enhance the ability of risk assessors to compare and combine results across studies. Inclusion of negative findings as well as positive findings also allows for more comprehensive understanding of the types of effects that have been assessed for a given chemical, allowing for improved comparison of results across species and study types. Taken together, the availability of new types of information and the inclusion of more detailed information in published reports will greatly enhance the utility of epidemiology data in human health risk assessments, and enhance our understanding of the contribution of environmental exposures to human health effects.

\section{DISCLAIMER}

The views expressed in this paper are those of the authors and do not necessarily reflect the views or policies of the U.S. Environmental Protection Agency.

\section{REFERENCES}

[1] NRC, Risk assessment in the federal government: Managing the process, Washington DC: National Research Council 1983; Available from: http://www.nap.edu/openbook.php?isbn=0309033 497

[2] USEPA, Risk Assessment Principles \& Practices (Office of the Science Advisor Staff Paper), Washington DC: Environmental Protection Agency 2004; Available from: http://www.epa.gov/OS A/pdfs/ratf-final.pdf

[3] EC, European Chemicals Bureau Technical Guidance Document on Risk Assessment, European Communities 2003; Available from: http://ecb.jrc.ec.europa.eu/documents/TECHNICAL_GUIDANCE_ DOCUMENT/EDITION_2/tgdpart2_2ed.pdf

[4] OECD, The OECD Environmental Risk Assessment Toolkit: Tools for Environmental Risk Assessment and Management, Organization for Economic Cooperation and Development 2010; Available from: http://www.oecd.org/document/54/0,3343,en_264 9_34373_44909430_1_1_1_1,00.html

[5] USEPA, Guidelines for mutagenicity risk assessment, Federal Register 51 (185) 34006-34012, 24 September 1986 Washington DC, Environmental Protection Agency, 1986; Available from: http://cfpub.epa.gov/ncea/cfm/recordisplay.cfm?deid=23160

[6] USEPA. Guidelines for Developmental Toxicity Risk Assessment, Risk Assessment Forum. Washington DC: Environmental 
Protection Agency 1991; Available from: http://cfpub.epa.gov/ ncea/cfm/recordisplay.cfm?deid $=23162$

[7] USEPA. Guidelines for carcinogen risk assessment, Federal Register 70 (66) 17765-17817, 7 April 2005. Washington DC: Environmental Protection Agency 1996; Available from: http://www.epa.gov/raf/publications/pdfs/propcra_1996.pdf

[8] USEPA. Guidelines for Reproductive Toxicity Risk Assessment, Risk Assessment Forum, Federal Register 61 (212) 56274-56322, 31 October 1996. Washington DC: Environmental Protection Agency 1996.

[9] USEPA. A Review of the Reference Dose and Reference Concentration Processes. Washington DC: Environmental Protection Agency 2002; Available from: http://cfpub.epa.gov/ ncea/cfm/reco rdisplay.cfm?deid $=55365$

[10] USEPA. Guidelines for carcinogenic risk assessment, Risk Assessment Forum, Federal Register 70 (66) 17765-17817, 7 April 2005. Washington DC: Environmental Protection Agency 2005a; Available from: http://www.epa.gov/iris/backgrd.htm

[11] USEPA, Supplemental guidance for assessing susceptibility from early-life exposure to carcinogens. Washington DC: Risk Assessment Forum Environmental Protection Agency 2005b; Available from: http://www.epa.gov/iris/backgrd.htm

[12] NRC. Science and Judgement in Risk Assessment. Washington DC: National Research Council 1994; Available from: http://www.nap.edu/openbook.php?record_id=2125

[13] NRC, Science and Decisions:Advancing Risk Assessment, National Research Council, Washington DC, 2009; Available from: http://www.nap.edu/catalog.php?record_id=12209

[14] Persad AS, Cooper GS. Use of epidemiologic data in Integrated Risk Information System (IRIS) assessments. Toxicol Appl Pharmacol 2008; 233: 137-45.

[15] Russell M, Gruber M. Risk assessment in environmental policymaking. Science 1987; 236: 286-90.

[16] Samet JM, Schnatter R, Gibb H. Epidemiology and risk assessment. Am J Epidemiol 1998; 148: 929-36.

[17] Hertz-Picciotto I. Epidemiology and quantitative risk assessment: a bridge from science to policy. Am J Public Health 1995; 85: 48491.

[18] Swaen GM. The use of epidemiological data in risk assessment. Regul Toxicol Pharmacol 1988; 8: 422-30.

[19] Swaen GM. A framework for using epidemiological data for risk assessment. Hum Exp Toxicol 2006; 25: 147-55.

[20] Steenland K, Thun M. Interaction between tobacco smoking and occupational exposures in the causation of lung cancer. J Occup Med 1986; 28: 110-8.

[21] Marsden CD. Parkinson's disease. Postgrad Med J 1992; 68: 53843.

[22] Bove J, Prou D, Perier C, et al. Toxin-induced models of Parkinson's disease. NeuroRx 2005; 2: 484-94.

[23] Sherer TB, Richardson JR, Testa CM, et al. Mechanism of toxicity of pesticides acting at complex I: relevance to environmental etiologies of Parkinson's disease. J Neurochem 2007; 100: 1469-79.

[24] Cory-Slechta DA. Studying toxicants as single chemicals: does this strategy adequately identify neurotoxic risk? Neurotoxicology 2005; 26: 491-510.

[25] Xiong N, Huang J, Zhang Z, et al. Stereotaxical infusion of rotenone: a reliable rodent model for Parkinson's disease. PLoS One 2009; 4: e7878.

[26] Brown TP, Rumsby PC, Capleton AC, et al. Pesticides and Parkinson's disease--is there a link? Environ Health Perspect 2006; 114: 156-64.

[27] Kamel F, Hoppin JA. Association of pesticide exposure with neurologic dysfunction and disease. Environ Health Perspect 2004; 112: 950-8.

[28] Gatto NM, Cockburn M, Bronstein J, et al. Well-water consumption and Parkinson's disease in rural California. Environ Health Perspect 2009; 117: 1912-8.
[29] Costello S, Cockburn M, Bronstein J, et al. Parkinson's disease and residential exposure to maneb and paraquat from agricultural applications in the central valley of California. Am J Epidemiol 2009; 169: 919-26.

[30] Ritz BR, Manthripragada AD, Costello S, et al. Dopamine transporter genetic variants and pesticides in Parkinson's disease. Environ Health Perspect 2009; 117: 964-9.

[31] Firestone JA, Smith-Weller T, Franklin G, et al. Pesticides and risk of Parkinson disease: a population-based case-control study. Arch Neurol 2005; 62: 91-5.

[32] Kamel F, Tanner C, Umbach D, et al. Pesticide exposure and selfreported Parkinson's disease in the agricultural health study. Am J Epidemiol 2007; 165: 364-74

[33] Petrovic P, Kalso E, Petersson KM, et al. Placebo and opioid analgesia-- imaging a shared neuronal network. Science 2002; 295 $1737-40$

[34] Hancock DB, Martin ER, Mayhew GM, et al. Pesticide exposure and risk of Parkinson's disease: a family-based case-control study. BMC Neurol 2008; 8: 6 .

[35] Payami H, Zareparsi S. Genetic epidemiology of Parkinson's disease. J Geriatr Psychiatry Neurol 1998; 11: 98-106.

[36] USEPA. OPPTS Harmonized Test Guidelines. Series 870 Health Effects. Washington DC: Environmental Protection Agency 1998; Available from: http://www.epa.gov/ocspp/pubs/frs/publicat ions/Test_Guidelines/series870.htm

[37] Eaton DL, Daroff RB, Autrup H, et al. Review of the toxicology of chlorpyrifos with an emphasis on human exposure and neurodevelopment. Crit Rev Toxicol 2008; 38 (Suppl) 2: 1-125.

[38] Slotkin TA. Cholinergic systems in brain development and disruption by neurotoxicants: nicotine, environmental tobacco smoke, organophosphates. Toxicol Appl Pharmacol 2004; 198: 132-51.

[39] Young JG, Eskenazi B, Gladstone EA, et al. Association between in utero organophosphate pesticide exposure and abnormal reflexes in neonates. Neurotoxicology 2005; 26: 199-209.

[40] Rauh VA, Garfinkel R, Perera FP, et al. Impact of prenatal chlorpyrifos exposure on neurodevelopment in the first 3 years of life among inner-city children. Pediatrics 2006; 118: e1845-59.

[41] Whyatt RM, Rauh V, Barr DB, et al. Prenatal insecticide exposures and birth weight and length among an urban minority cohort Environ Health Perspect 2004; 112: 1125-32.

[42] Eskenazi B, Marks AR, Bradman A, et al. Organophosphate pesticide exposure and neurodevelopment in young MexicanAmerican children. Environ Health Perspect 2007; 115: 792-8.

[43] Engel SM, Berkowitz GS, Barr DB, et al. Prenatal organophosphate metabolite and organochlorine levels and performance on the Brazelton Neonatal Behavioral Assessment Scale in a multiethnic pregnancy cohort. Am J Epidemiol 2007; 165: 1397404.

[44] USEPA. September 16 - 19, 2008: The Agency's Evaluation of the Toxicity Profile of Chlorpyrifos, Washington DC: Environmental Protection Agency 2008; Available from: http://www.epa.gov/sci poly/sap/meetings/2008/091608_mtg.htm\#frn

[45] Tseng WP. Effects and dose-response relationships of skin cancer and blackfoot disease with arsenic. Environ Health Perspect 1977; 19: 109-19.

[46] Tseng WP, Chu HM, How SW, et al. Prevalence of skin cancer in an endemic area of chronic arsenicism in Taiwan. J Natl Cancer Inst 1968; 40: 453-63.

[47] USEPA, Special Report on Ingested Inorganic Arsenic; Skin Cancer, Nutritional Essentiality Risk Assessment Forum, Environmental Protection Agency, 1988; Available from: http://cfpub.epa. gov/ncea/cfm/recordisplay.cfm?deid=20866

[48] Furst A. A new look at arsenic carcinogenesis, in: W. Lederer R. Fensterheim Eds Aresenic: Industrial, Biomedical, and Environmental Perspectives, New York: Van Nostrand Reinhold 1983; pp. p. 151-63. 